\title{
Efficacy and safety of bioresorbable scaffolds in patients with coronary bifurcation lesions: A systematic review and meta-analysis
}

\author{
Xi-Ying Liang ${ }^{1 *} \odot$, Yan $\mathrm{Li}^{1 *}$, Wen-Jiao Zhang ${ }^{1 *}$, Xuan Qiao ${ }^{1 *}$, \\ Rong-Rong Yang ${ }^{1}$, Zhi-Lu Wang ${ }^{2}(-)$
}

${ }^{1}$ The First Clinical Medical College of Lanzhou University, Lanzhou, Gansu, China

${ }^{2}$ Department of Cardiology, The First Hospital of Lanzhou University, Lanzhou, Gansu, China

\begin{abstract}
Background: Bioresorbable scaffolds (BRS) were considered to be beneficial for coronary bifurcation lesions regarding the avoidance of lateral branch opening incarceration after complete absorption. However, data is limited in this setting. The aim of this meta-analysis was to evaluate the short (6-month) and medium-term (1-year) outcomes of BRS in patients with coronary bifurcation lesions.

Methods: PubMed, EMBASE, Web of Science, Cochrane library databases were searched to find the studies of BRS implantation in patients with coronary bifurcation lesions. The effective outcome was target lesion revascularization. The safety outcomes included major adverse cardiovascular events, target vessel revascularization, myocardial infarction, definite or probable scaffold thrombosis, and cardiac death.

Results: A total of 1204 patients involved in 12 studies were included. The pooled estimate rate of target lesion revascularization as efficacy outcome was highly consistent between 6-month and 1-year follow-up, which was 4.74\% (95\% CI 2.36-9.54\%, $\left.I^{2}=41.5 \%, p=0.14\right)$ and $4.37 \%$ (95\% CI $\left.3.05-5.69 \%, I^{2}=4.6 \%, p=0.39\right)$. The pooled estimated rate of major adverse cardiovascular events as safety outcome was $5.50 \%$ and $7.31 \%$ for both 6-month and 1-year follow-up. The pooled estimated rate of target vessel revascularization, myocardial infarction, definite or probable scaffold thrombosis, and cardiac death at 1-year follow-up was 5.92\%, 2.52\%, 1.69\%, and 0.42\%.

Conclusions: The application of BRS for coronary bifurcation lesions is acceptable in efficacy outcome, but the high rate of scaffold thrombosis remains of concern (Registered by PROSPERO, CRD42019140341). (Cardiol J, 2022; 29, 4: 563-573)

Key words: bioresorbable scaffolds, coronary bifurcation lesions, percutaneous coronary intervention, meta-analysis
\end{abstract}

\section{Introduction}

Bifurcation lesions are common complex coronary artery lesions, accounting for $15-20 \%$ of percutaneous coronary intervention (PCI), and are also one of the most challenging lesions in interventional cardiology from the point of view for procedural success rate and long-term cardiac events [1]. Drug-eluting stents (DES) are currently recommended for the treatment of coronary bifurcation lesions [2]. However, the DES can lead to inflammation, poor adherence and impaired vasodilation, which may also limit the possibility of re-intervention after permanent implantation. In

Address for correspondence: Dr. Zhilu Wang, Department of Cardiology, The First Hospital of Lanzhou University, No. 1, Donggang West Road, Chengguan District, Lanzhou, Gansu 730000, China, tel: +86 13893207195, e-mail: wangzhl@lzu.edu.cn Received: 2.12 .2020 Accepted: 29.03.2021 Early publication date: 9.04 .2021

*These authors contributed equally to this work.

This article is available in open access under Creative Common Attribution-Non-Commercial-No Derivatives 4.0 International (CC BY-NC-ND 4.0) license, allowing to download articles and share them with others as long as they credit the authors and the publisher, but without permission to change them in any way or use them commercially. 
addition, the risks of late stent thrombosis and instent restenosis are still the major factors affecting the efficacy of coronary artery disease especially coronary bifurcation lesions [3, 4].

The emergence of bioresorbable scaffolds (BRS), which can be traced back to 1980 s may be conducive to this problem with the following advantages: shorter arterial healing time than DES implantation, late lumen expansion can reduce the risk of restenosis and avoidance of long-term jailing for side branch stent after complete resorption of scaffold wire within 2-3 years following implantation [4]. Meanwhile, it is believed that restoration of vascular patency may be more important for treatment of coronary bifurcation lesions in the absence of permanent implants [5]. Additional potential advantages include easier imaging (cardiac computed tomography or magnetic resonance imaging) and increased lumen area [6].

The applications of BRS are being extended to more complex lesions in the real-world study. However, there is still a lack of randomized controlled trials for coronary bifurcation lesions; the available data are limited to observational studies of conclusion conflict $[7,8]$. The current expert consensus only provided a limited recommendation for the application of this new technology in coronary bifurcation lesions [1, 2, 9]. Given the advantages and clinical setting of BRS, a hypothesis to be beneficial for patients with coronary bifurcation lesions was established. Therefore, this systematic review and meta-analysis was designed to evaluate the efficacy and safety of BRS for the short- (6-month) and medium-term (1-year) in patients with those.

\section{Methods}

\section{Search strategy}

The present systematic review and metaanalysis was performed according to the Preferred Reporting Items for Systematic Reviews and Meta-Analyses (PRISMA) consensus statement [10] and the Meta-analysis of Observational Studies in Epidemiology (MOOSE) consensus statement [11]. PubMed, EMBASE, Web of Science, Cochrane library databases were searched with the following keywords: "coronary bifurcation lesion*" AND "bioresorbable scaffold*" OR "bioresorbable vascular stent*" (Suppl. File S1) with no language restrictions from inception to September 21, 2019. The references from relevant articles were scanned for additional studies not identified in the initial database search. An automated reminder from the PubMed was set up to track the latest publications.
All reports were independently screened by two investigators (X.Y. Liang and Y. Li) to determine whether they met the inclusion criteria and any disagreement was resolved by consultation. The study protocol was registered in PROSPERO (CRD42019140341).

\section{Selection criteria}

The inclusion criteria were as follows: (1) patients with at least one coronary bifurcation lesion (de novo bifurcation lesion involving a side-branch $\geq 2 \mathrm{~mm}$ by visual estimation in diameter); (2) at least 1 BRS implanted; (3) at least 9 patients were included in the study; (4) trials reported clinical outcomes for at least 6 months; (5) included at least 1 clinical outcome, such as target lesion revascularization (TLR), major adverse cardiovascular events (MACE), target vessel revascularization (TVR), myocardial infarction (MI), definite or probable scaffold thrombosis (ST) or cardiac death.

The exclusion criteria included: (1) experimental studies on animals; (2) case report, conference abstract, review or expert opinions; (3) incomplete description (no complete report for patient characteristics and clinical outcomes of coronary bifurcation lesions); (4) duplicate publication or duplicate studies (if duplicate studies were identified, only the most exhaustive and recent reports were retained).

\section{Data extraction}

Baseline characteristics, lesion and procedural characteristics for patients, as well as numbers of events, were independently extracted from the original publications by 4 investigators (X.Y. Liang, Y. Li, W.J. Zhang, and X. Qiao). Divergences were resolved through discussions with the third party (Z.L. Wang).

\section{Outcomes and definitions}

The effective outcome was TLR, defined as any repeated PCI or coronary artery bypass grafting (CABG) for the segment of previously treated or in the adjacent $5 \mathrm{~mm}$. The safety outcomes were MACE, TVR, any MI, definite or probable ST, and cardiac death. The MACE was defined according to the definitions of the original trials. The TVR was defined as repeat PCI or CABG in the target vessel. The MI was defined according to the universal definition [12]. The definite or probable ST was classified according to the Academic Research Consortium criteria [13]. Deaths that could not be attributed to another cause was regarded as cardiac death. 


\section{Quality assessment}

Depending on the type of study included, the Newcastle-Ottawa scale checklist [14] and the Joanna Briggs Institute Critical Appraisal Checklist for Case Series (https://joannabriggs.org/research/ /critical-appraisal-tools.html) were used to assess the quality of non-randomized studies and case series. The quality of all studies was independently evaluated by 2 investigators (X.Y. Liang and Y. Li) and any dispute was settled by a third party through negotiations (Z.L. Wang). Furthermore, GRADE-profiler 3.2.2 was performed to appraise the quality of the evidence as high, moderate, low or very low grades [15]. As analyses were based on previously published studies, ethical approval and patient consent were not required.

\section{Statistical analysis}

Statistical analysis was performed on the pooled data of all included studies. The metaprop command was used to calculate combined rate with $95 \%$ confidence interval $(95 \% \mathrm{CI})$. The normality test was employed for untransformed proportion (PRAW) and the rate transformed by Natural Logar Transformed Proportion, Logit Transformed Proportion, Arcsine Transformed Proportion or Freeman-Tukey Double Arcsine Transformed before the metaprop analysis, and the method close to the normal distribution was selected according to the results. Meta-analyses of dichotomous variables were expressed as odds ratio (OR) and 95\% CI. Heterogeneity was assessed by the Higgins $\mathrm{I}^{2}$ test, and random-effects model was applied to calculate the statistic effects. Publication bias was evaluated by visual estimation of funnel plots and the Egger's test at the $p<0.05$ level of significance. The sensitivity analysis was performed by omitting one study from the analysis at a time. The subgroup analysis was utilized to explore the effect of different conditions (acute coronary syndrome [ACS] or not, diabetes mellitus [DM] or not) on outcomes. The p-value threshold of two-tailed significance was 0.05 . Analyses were performed with R 3.6.1 (R Foundation for Statistical Computing, Vienna, Austria and Stata SE 14.0 (StataCorp LP, College Station, Texas).

\section{Results}

\section{Search results and characteristics}

At first, 532 articles were retrieved, which were reduced to 144 studies after screening the title and abstract. Finally, 12 studies are included in the quantitative synthesis after excluding most unrelated studies (Fig. 1) [7, 8, 16-25]. Among them, the Desolve 150 BRS (Elixir, US) was used in one study [22], while others used the second-generation everolimus-eluting bioresorbable vascular scaffolds (ABSORB; Abbott Vascular, Santa Clara, CA, USA). A total of 1,204 patients met the inclusion criteria and were included, of whom 1,014 (84.2\%) were male patients, 573 (47.6\%) patients with ACS and 369 (30.7\%) patients with DM in Table 1 . The lesion and procedure features are listed in Table 2 . The quality assessments for studies are reported in Supplementary Tables S1 and S2. GRADE quality assessment is provided in Supplementary Table S3.

\section{The effective outcome}

Five studies provided data on the effective outcome of TLR at 6-month follow-up and the pooled estimate rate of TLR is $4.74 \%$ (95\% CI 2.36-9.54\%, $\mathrm{I}^{2}=41.5 \%, \mathrm{p}=0.14$; Fig. 2). A significant asymmetry was observed in the funnel plot by visual estimation (Suppl. Fig. S1a). However, the publication bias was not detected by the Egger's test $(\mathrm{p}=0.81)$. The sensitivity analysis shows that the pooled estimate rate was reduced from $4.74 \%$ to $3.53 \%$ and heterogeneity of the remaining studies had changed significantly $\left(\mathrm{I}^{2}=0 \%\right)$ when a study was omitted (Suppl. Fig. S2). The effective outcome for 1 year was followed-up in 8 studies, which shows that the pooled estimate rate of TLR was $4.37 \%\left(95 \%\right.$ CI $\left.3.05-5.69 \%, \mathrm{I}^{2}=4.6 \%, \mathrm{p}=0.39\right)$ (Fig. 2). For this outcome, no asymmetry is identified in the funnel plot by visual estimation (Suppl. Fig. S1b), and no significant publication bias was found by the Egger's test $(\mathrm{p}=0.11)$.

\section{The safety outcomes}

The safety outcomes are presented (Fig. 3). The pooled estimate rate of MACE was $5.50 \%$ $\left(95 \%\right.$ CI $\left.0.56-10.45 \%, \mathrm{I}^{2}=41.2 \%, \mathrm{p}=0.15\right)$ at 6-month follow-up and 7.31\% (95\% CI 4.69-9.92\%, $\left.\mathrm{I}^{2}=36.9 \%, \mathrm{p}=0.16\right)$ at 1 -year follow-up. The sensitivity analysis shows that the pooled estimate rate of MACE at 6-month follow-up had decreased from $5.50 \%$ to $3.89 \%$ and heterogeneity of the remaining studies had changed significantly $\left(\mathrm{I}^{2}=0 \%\right)$ after omitting one study (Suppl. Fig. S2b). The initial pooled estimate rates of TVR and MI at 1-year follow-up were $5.92 \%$ (95\% CI 3.62-8.22\%, $\left.\mathrm{I}^{2}=15.5 \%, \mathrm{p}=0.31\right)$ and $2.52 \%(95 \%$ CI $1.32-$ $\left.-3.73 \%, \mathrm{I}^{2}=28.6 \%, \mathrm{p}=0.19\right)$. The pooled estimate rate was $1.69 \%\left(95 \%\right.$ CI $0.80-2.58 \%, \mathrm{I}^{2}=0 \%$, $\mathrm{p}=0.49)$ for the definite or probable ST and $0.42 \%\left(95 \%\right.$ CI $\left.0-0.95 \%, \mathrm{I}^{2}=0 \%, \mathrm{p}=0.97\right)$ for 


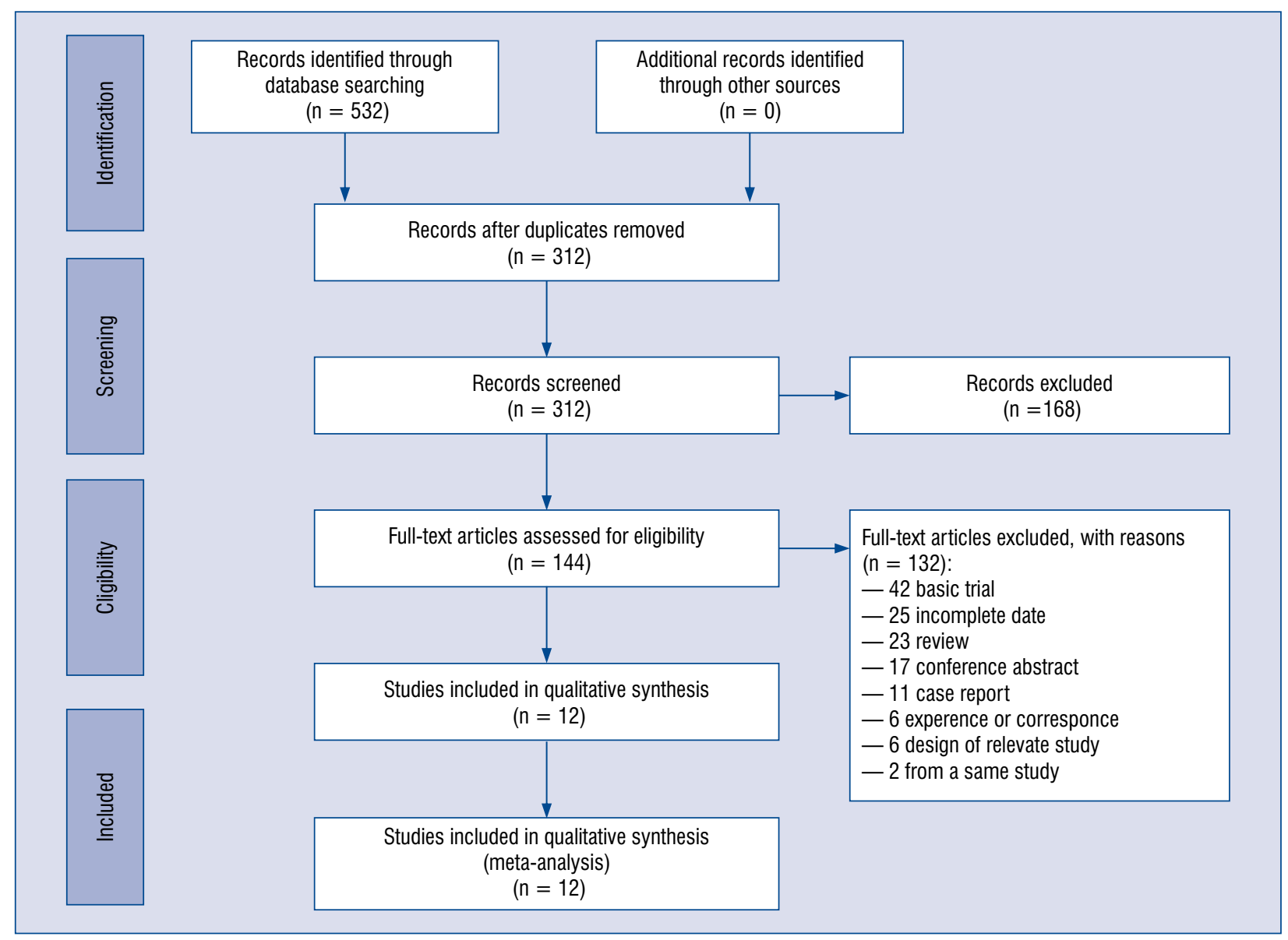

Figure 1. Flow chart of study selection.

cardiac death without publication bias at 1-year follow-up. The effects of the simple and complex strategies on the definite or probable ST are extracted and analyzed in 5 studies, which shows that there was no statistical significance between the simple and complex strategies $(\mathrm{OR}=0.86,95 \% \mathrm{CI}$ $0.248-2.624 ; \mathrm{I}^{2}=0 \%, \mathrm{p}=0.981$; Fig. 4).

\section{Subgroup analysis}

Two subgroup analyses were performed according to the median proportion of patients with ACS and DM in the 12 studies included. The ACS was stratified according to whether the median proportion exceeded $40.35 \%$, the DM was stratified according to whether the median proportion exceeded $28.85 \%$. The results showed that the pooled estimate rate of MI, definite or probable ST and cardiac death was increased in patients with ACS and DM (3.82\% vs. $1.54 \%, 2.52 \%$ vs. $1.28 \%$ and $0.66 \%$ vs. $0.35 \%$ for the subgroup of ACS vs. non-ACS, respectively; $4.04 \%$ vs. $2.03,3.01 \%$ vs. $1.54 \%$ and $1.06 \%$ vs. $0.40 \%$ for the subgroup of DM vs. non-DM, respectively), which increase nearly or more than twice (Suppl. Figs. S3 and S4). The effect on TLR, MACE and TVR is not significant for both ACS and DM subgroups (Suppl. Figs. S5 and S6).

\section{Discussion}

According to available research, this is the first systematic review and meta-analysis involving the application of BRS for patients with coronary bifurcation lesions. The major findings are as follows: (1) the pooled estimated rate of TLR as the effective outcome of BRS for patients with those is acceptable at short- and medium-term follow-up; (2) a majority of the safety outcomes (MACE, TVR, MI and cardiac death) have reached the safety effect size; (3) the rate of definite or probable ST remains a concern. However, these findings are only based on observational studies with very low GRADE quality. Therefore, this estimate is very uncertain, and additional 


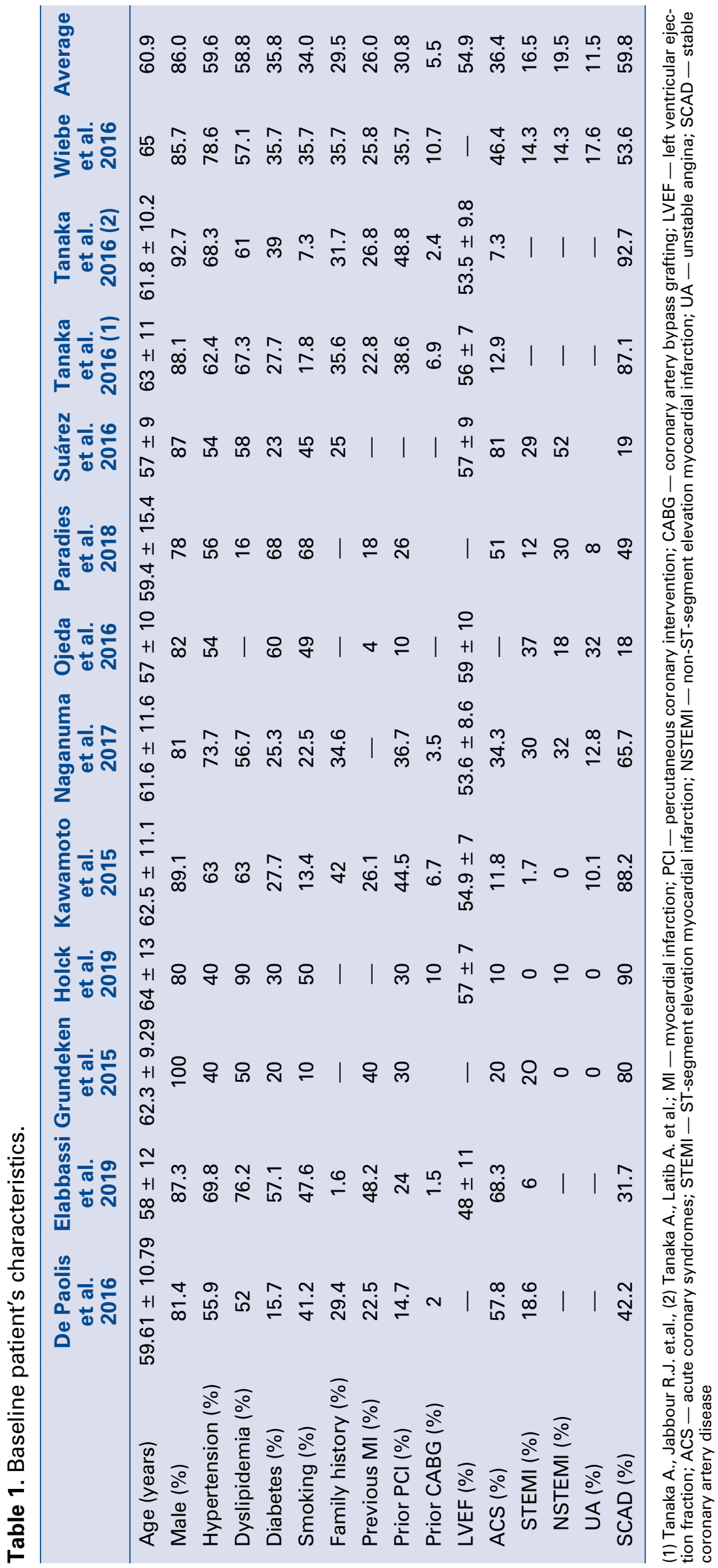




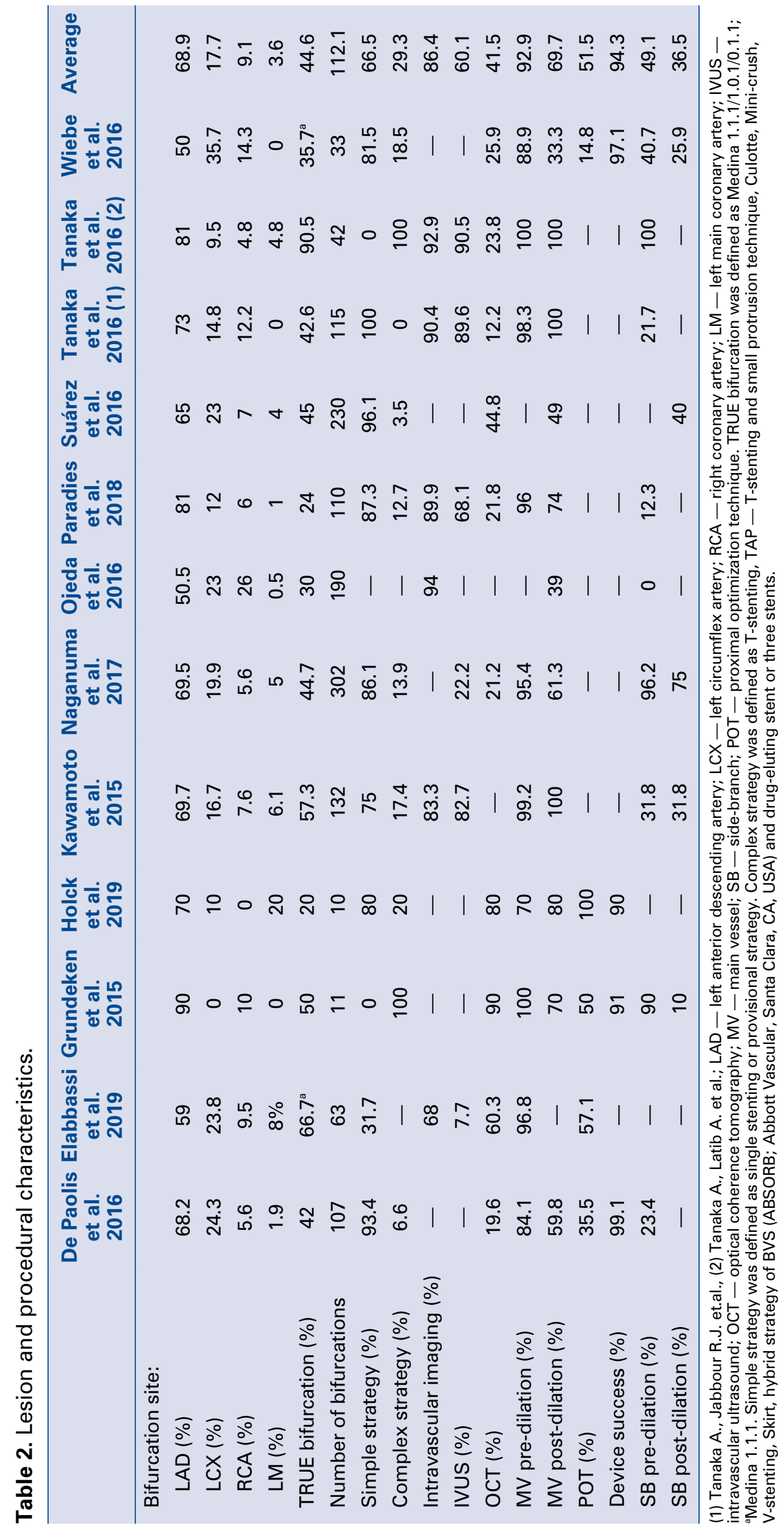




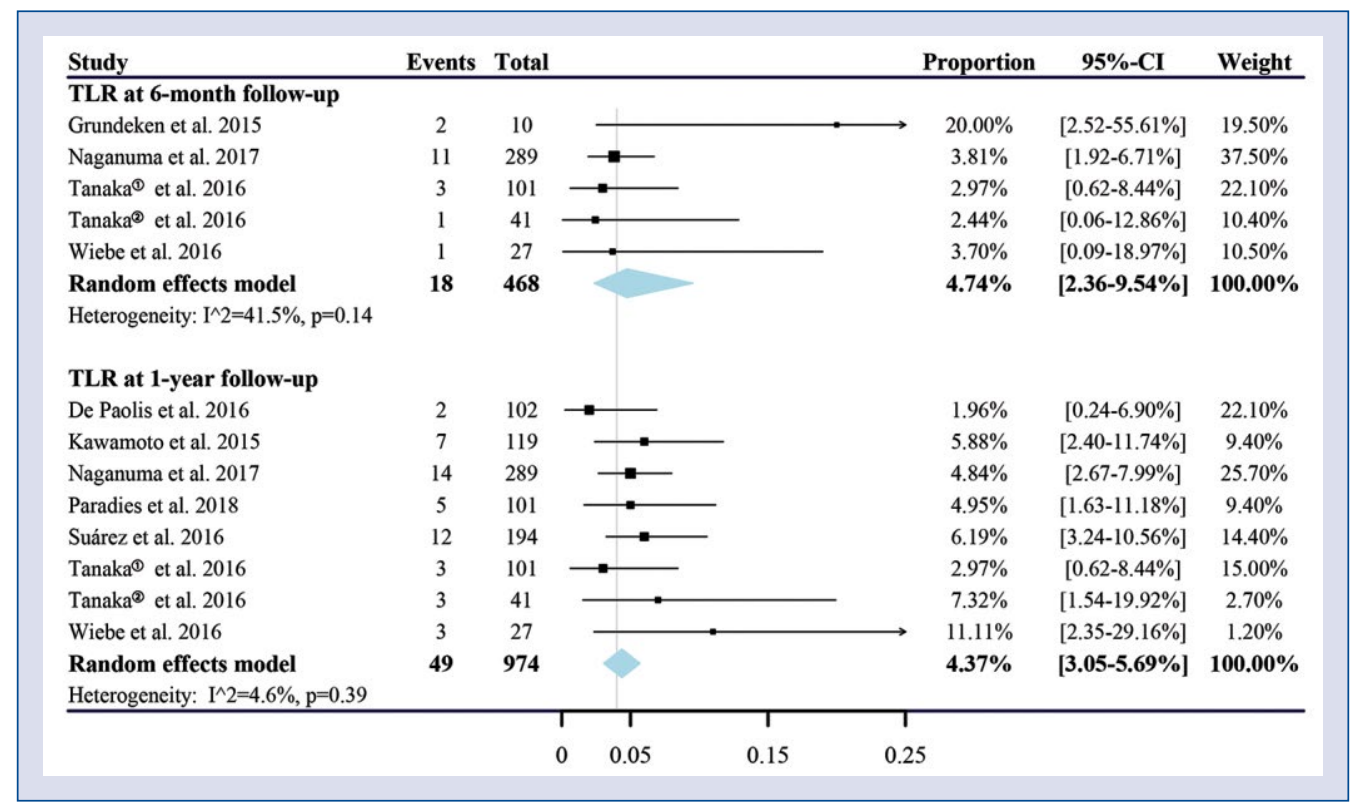

Figure 2. Primary endpoints for studies included; $\mathrm{Cl}$ - confidence interval; TLR — target lesion revascularization.

randomized trials are required to provide higher quality evidence.

The BRS has the characteristic of complete absorption and is known as the fourth revolution in the history of coronary intervention, which changed the long-term problem of permanent implantation and achieved complete revascularization/restoration of vascular patency. The Igaki-Tamai stent was the first biodegradable stent to be included in human trials with long-term ( $>10$ year) clinical outcomes and intravascular ultrasound data, which had acceptable MACE and ST rates without stent recoil and vessel remodeling [26]. It revealed a promising early result. More than 10 BRS with different backbone, different coating drug dose, different strut thickness, different vessel coverage area and different complete resorption time have been tested in clinical practice to date. However, these scaffolds are still in clinical research and lack of powerful evidence. Most experience came from bioresorbable vascular scaffolds (BVS) of Abbott, whereas poor clinical results for ST and low market share led to its delisting in 2017. This appears to place a veil over the use of BRS. However, two recently published randomized controlled trials showed optimistic results. One was the ABSORB IV trial [27], which showed that BVS resulted in non-inferior rates of target lesion failure and angina pectoris compared with metallic DES, another was the implanted NeoVas BRS that indicted non-inferior to metallic DES for angiographic in-segment late loss and clinical outcomes [28]. The NeoVas BRS is a new generation BRS, which could elute sirolimus from a poly-D, 1-lactide coating. Nonetheless, these do not include coronary bifurcation lesions.

Currently, there is limited evidence to investigate the clinical results of BRS in coronary bifurcation lesions, although Stankovic and Lassen [5] believed that BRS might better provide profit in this specific lesion. The incidence of TLR and majority safety outcomes for BRS was acceptable compared with first- and second-generation DES in coronary bifurcation lesions 29, 30]. Another notable problem is that the results were obtained on the basis that the side branch was more than at least $2 \mathrm{~mm}$, regardless of intervention or not. The BRS was designed with increased strut thickness, which is easier to protrude into the side branch and occlude it. Therefore, the coronary bifurcation lesions should be selected cautiously when BRS was used, which were supported by studies from Muramatsu et al. [31] and Ojeda et al. [21]. The rate of MACE was significantly lower at 1-year follow-up in this meta-analysis than that of the second-generation DES (6.91\% vs. $12.1 \%)$, which might be due to different event definitions rather than an obvious advantage. It should also be emphasized that the incidence of definite or probable ST implanted BRS was significantly higher than that of second-generation DES ( $1.61 \%$ vs. $0.7 \%$ with $1^{\text {st }}$-stent, $1.4 \%$ with $2^{\text {nd }}$-stent). The same results were drawn in simple lesions, which needs to be addressed to reduce 


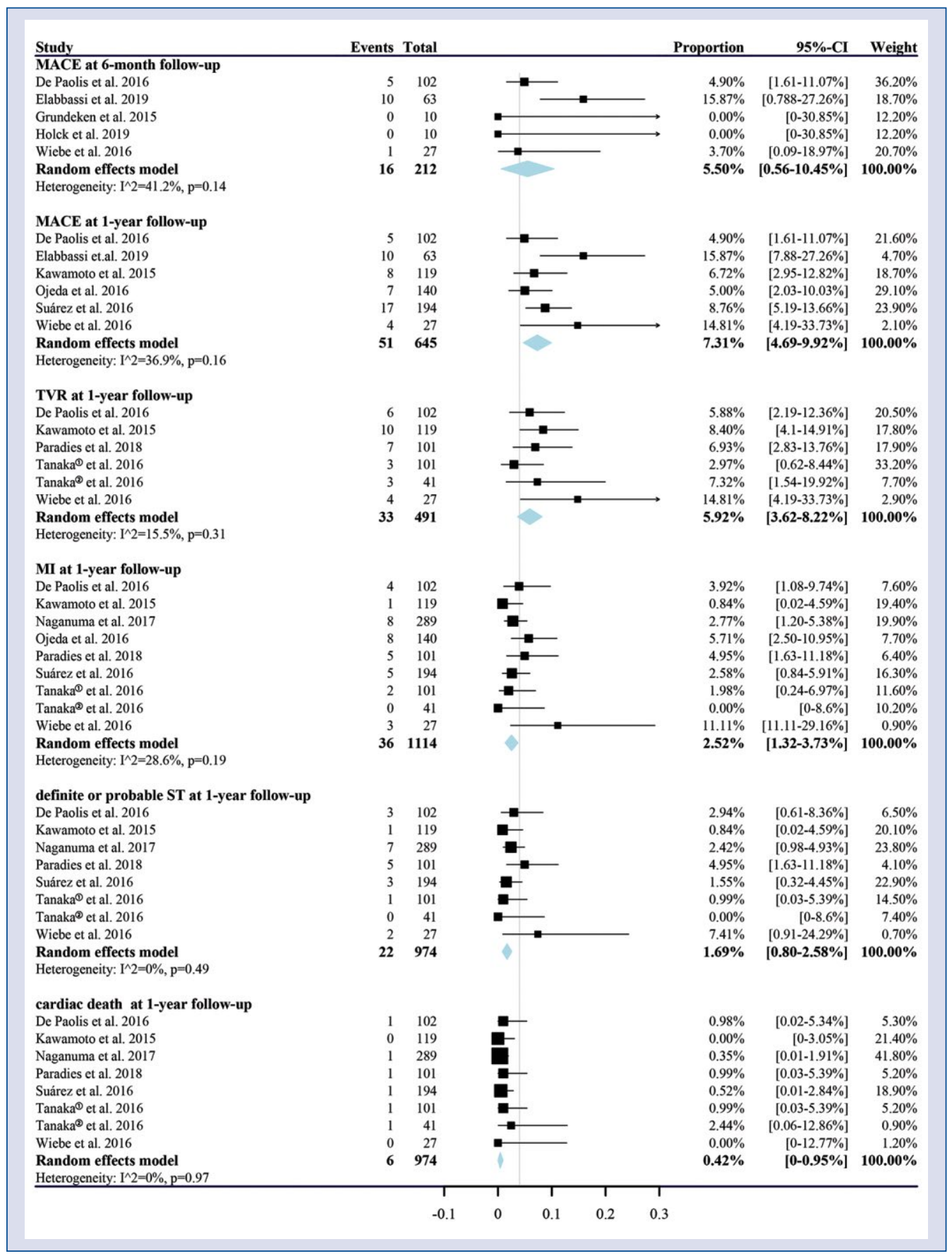

Figure 3. Secondary endpoints for studies included; $\mathrm{Cl}$ — confidence interval; MACE — major adverse cardiac events; $\mathrm{MI}$ - myocardial infarction; TVR — target vessel revascularization; ST — scaffold thrombosis.

the rate of definite or probable ST. First of all, this result can be improved by more appropriate lesion selection and standard implantation techniques (pre-dilation, sizing and post-dilation technique). In addition, the provisional strategy was recommended for bifurcation lesions according to present consensus and guidelines [1,2]. This study did not provide further recommendations for simple and complex strategies, but the simple strategy (single stent or provisional strategy) is obviously more popular. Previous research has shown that premature discontinuation of antiplatelet therapy exacerbated risk of scaffold thrombosis [8]. The 2018 ESC/EACTS Guidelines recommended that dual antiplatelet therapy should be considered for at least 12 months and up to the presumed full absorption of BRS [2]. Although the BRS was designed to reduce the duration of long-term an- 


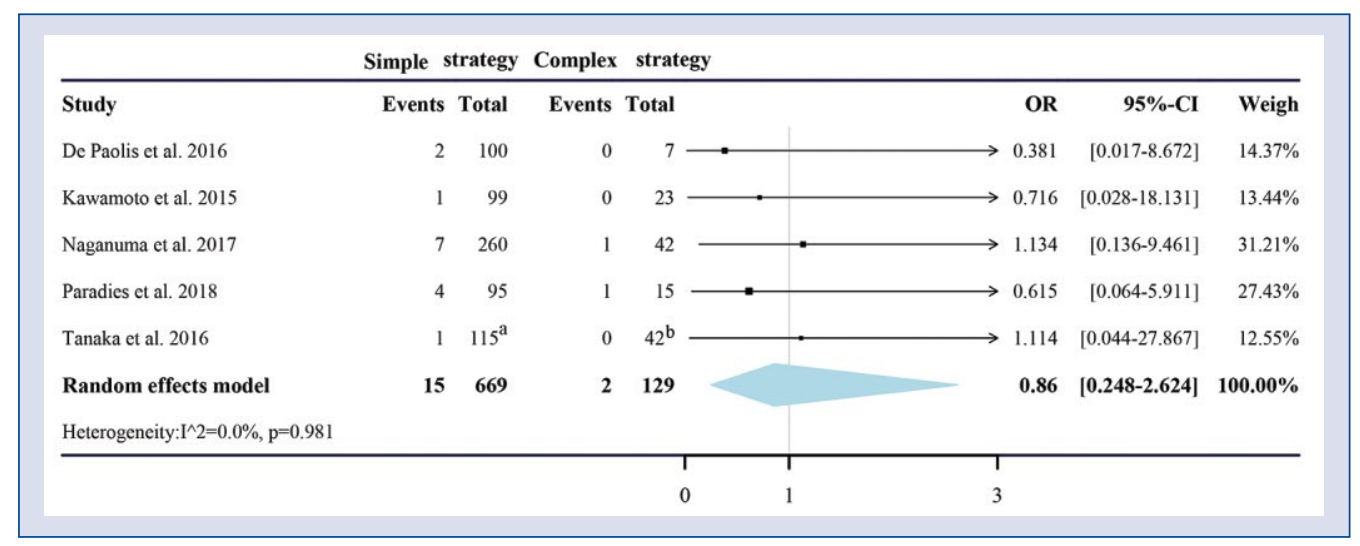

Figure 4. Effects of simple and complex strategies on definite or probable scaffold thrombosis for studies included ('Data from [24]; 'Data from [25]); Cl — confidence interval; OR - odds ratio.

tiplatelet therapy, this may occur after complete stent degradation. The result of a registry study, BVS LATE (NCT02939872), intended to evaluate the optimal duration of antiplatelet therapy after BVS implantation is to be expected. Preliminary subgroup analysis showed that ACS and DM were risk factors of partial safety outcomes, which were similar to the coronary bifurcation lesions substudy from GHOST EU Registry [20]. This suggests a more conservative approach to patient selection.

Nevertheless, the results must be interpreted cautiously before there is insufficient evidence to support them. Firstly, considering the difference of baseline characteristics and the BRS types, as well as varied definitions of clinical outcome, the event rates might be influenced. Secondly, most of these results were from European, and may require data from more regional and ethnic populations to determine whether the results can be extrapolated. In addition, these data were only observational studies from specific clinical centers which had better implantation technology and higher operation success rates. Furthermore, the proportion of intravascular imaging varies greatly, which has an important impact on of procedure and incidence of postoperative events, and is an important reason for the differences in the results of the studies. Therefore, it also needs to be evaluated by randomized controlled trials. Meanwhile, BRS should be implanted cautiously in patients with a high risk of bleeding who cannot tolerate dual antiplatelet therapy for 12 months. Furthermore, the quality of evidence and the strength of recommendation for the studies included were very low according to GRADE criteria, which was because of limita- tions of the single-arm observational study design, lack of indirect evidence from a control group, and inaccurate results due to small sample sizes. However, the purpose of this meta-analysis was to summarize the evidence, it should not be widely used in clinical practice without further evidence.

\section{Limitation of the study}

The limitations of this meta-analysis should be considered. Firstly, the studies included were single-arm or observational studies with small sample sizes, most of which were single-center data, which significantly decreased the level of evidence for study. Secondly, the absorbable scaffolds implanted in the meta-analysis were not uniform, with BVS dominating, and no magnesium - BRS were included. These differences may affect the results of study. Thirdly, the data of subgroup analysis were based on the median of patients with DM and ACS in the single-arm study, which was not supported by the specific evidence, the results should be interpreted carefully. Fourthly, due to the limitations of current clinical studies, the duration of dual antiplatelet therapy and the optimal strategy in this study had not been clearly explained. Lastly, longer-term results after the stents complete absorption have also not been reported.

\section{Conclusions}

This meta-analysis shows that the application of BRS for coronary bifurcation lesions is acceptable in efficacy outcome and most safety outcomes, but the high rate of ST remains a concern. The efficacy and safety of BRS on coronary bifurcation 
lesions should be explored in large-scale randomized controlled trials in the future.

\section{Conflict of interest: None declared}

\section{References}

1. Lassen JF, Holm NR, Banning A, et al. Percutaneous coronary intervention for coronary bifurcation disease: 11th consensus document from the European Bifurcation Club. EuroIntervention. 2016; 12(1): 38-46, doi: 10.4244/EIJV12I1A7, indexed in Pubmed: 27173860.

2. Neumann FJ, Sousa-Uva M, Ahlsson A, et al. ESC Scientific Document Group, ESC Scientific Document Group. Considerations for the choice between coronary artery bypass grafting and percutaneous coronary intervention as revascularization strategies in major categories of patients with stable multivessel coronary artery disease: an accompanying article of the task force of the $2018 \mathrm{ESC} / \mathrm{EACTS}$ guidelines on myocardial revascularization. Eur Heart J. 2019; 40(2): 204-212, doi: 10.1093/eurheartj/ ehy532, indexed in Pubmed: 30165435.

3. McFadden EP, Stabile E, Regar E, et al. Late thrombosis in drug-eluting coronary stents after discontinuation of antiplatelet therapy. Lancet. 2004; 364(9444): 1519-1521, doi: 10.1016/ S0140-6736(04)17275-9, indexed in Pubmed: 15500897.

4. Colleran R, Byrne RA. Bioresorbable vascular scaffolds in coronary bifurcation lesions: only in expert hands. Rev Esp Cardiol (Engl Ed). 2016; 69(6): 543-546, doi: 10.1016/j.rec.2016.03.011, indexed in Pubmed: 27157886.

5. Stankovic G, Lassen JF. When and how to use BRS in bifurcations? EuroIntervention. 2015; 11 Suppl V: V185-V187, doi: 10.4244/EIJV11SVA44, indexed in Pubmed: 25983163.

6. Iqbal J, Onuma Y, Ormiston J, et al. Bioresorbable scaffolds: rationale, current status, challenges, and future. Eur Heart J. 2014; 35(12): 765-776, doi: 10.1093/eurhearti/eht542, indexed in Pubmed: 24366915.

7. De Paolis M, Felix C, van Ditzhuijzen N, et al. Everolimuseluting bioresorbable vascular scaffolds implanted in coronary bifurcation lesions: Impact of polymeric wide struts on side-branch impairment. Int J Cardiol. 2016; 221: 656-664, doi: 10.1016/j. ijcard.2016.06.153, indexed in Pubmed: 27423087.

8. Wiebe J, Dörr O, Bauer T, et al. Everolimus-eluting bioresorbable scaffold implantation for the treatment of bifurcation lesions: Implications from early clinical experience during daily practice. Cardiovasc Revasc Med. 2016; 17(5): 313-317, doi: 10.1016/j. carrev.2016.03.011, indexed in Pubmed: 27085220.

9. Ormiston JA, Kassab G, Finet G, et al. Bench testing and coronary artery bifurcations: a consensus document from the European Bifurcation Club. EuroIntervention. 2018; 13(15): e1794-e1803, doi: 10.4244/EIJ-D-17-00270, indexed in Pubmed: 29131803.

10. Moher D, Liberati A, Tetzlaff J, et al. Preferred reporting items for systematic reviews and meta-analyses: the PRISMA statement. PLoS Medicine. 2009; 6(7): e1000097, doi: 10.1371/journal.pmed.1000097.

11. Stroup DF, Berlin JA, Morton SC, et al. Meta-analysis of observational studies in epidemiology: a proposal for reporting. Meta-analysis Of Observational Studies in Epidemiology (MOOSE) group. JAMA. 2000; 283(15): 2008-2012, doi: 10.1001/ jama.283.15.2008, indexed in Pubmed: 10789670.
12. Thygesen K, Alpert JS, Jaffe AS, et al. Third universal definition of myocardial infarction. Eur Heart J. 2012; 33(20): 2551-2567, doi: 10.1093/eurheartj/ehs184, indexed in Pubmed: 22922414.

13. Cutlip DE, Windecker S, Mehran R, et al. Academic Research Consortium. Clinical end points in coronary stent trials: a case for standardized definitions. Circulation. 2007; 115(17): 2344-2351, doi: 10.1161/CIRCULATIONAHA.106.685313, indexed in Pubmed: 17470709.

14. Stang A. Critical evaluation of the Newcastle-Ottawa scale for the assessment of the quality of nonrandomized studies in metaanalyses. Eur J Epidemiol. 2010; 25(9): 603-605, doi: 10.1007/ s10654-010-9491-z, indexed in Pubmed: 20652370.

15. Atkins D, Best D, Briss PA, et al. GRADE Working Group. Grading quality of evidence and strength of recommendations. BMJ. 2004; 328(7454): 1490, doi: 10.1136/bmj.328.7454.1490, indexed in Pubmed: 15205295.

16. Elabbassi W, Chowdhury MA, Hatala R. Use of Bioresorbable Vascular Scaffold technology in treating coronary bifurcation lesions: A report about long-term clinical results and review of available literature. Bratisl Lek Listy. 2019; 120(8): 545-551, doi: 10.4149/BLL_2019_088, indexed in Pubmed: 31379174.

17. Grundeken MJ, Hassell ME, Kraak RP, et al. Treatment of coronary bifurcation lesions with the Absorb bioresorbable vascular scaffold in combination with the Tryton dedicated coronary bifurcation stent: evaluation using two- and three-dimensional optical coherence tomography. EuroIntervention. 2015; 11(8): 877-884, doi: 10.4244/EIJY14M08_15, indexed in Pubmed: 25169592.

18. Holck EN, Fox-Maule C, Barkholt TO, et al. Procedural findings and early healing response after implantation of a self-apposing bioresorbable scaffold in coronary bifurcation lesions. Int J Cardiovasc Imaging. 2019; 35(7): 1199-1210, doi: 10.1007/s10554019-01537-5, indexed in Pubmed: 31053981.

19. Kawamoto H, Latib A, Ruparelia N, et al. Clinical outcomes following bioresorbable scaffold implantation for bifurcation lesions: Overall outcomes and comparison between provisional and planned double stenting strategy. Catheter Cardiovasc Interv. 2015; 86(4): 644-652, doi: 10.1002/ccd.26045, indexed in Pubmed: 26013748.

20. Naganuma T, Colombo A, Lesiak M, et al. Bioresorbable vascular scaffold use for coronary bifurcation lesions: A substudy from GHOST EU registry. Catheter Cardiovasc Interv. 2017; 89(1): 47-56, doi: 10.1002/ccd.26634, indexed in Pubmed: 27414021.

21. Ojeda S, Pan M, Suárez de Lezo J, et al. Patency of coronary side branches covered by an everolimus-eluting bioresorbable vascular scaffold: clinical outcomes and computed tomography scan follow-up. EuroIntervention. 2016; 11(11): e1283-e1290, doi: 10.4244/EIJV11111A250, indexed in Pubmed: 26865446.

22. Paradies V, Vlachojannis GJ, Royaards KJ, et al. Angiographic and midterm outcomes of bioresorbable vascular scaffold for coronary bifurcation lesions. Am J Cardiol. 2018; 122(12): 2035-2042, doi: 10.1016/j.amjcard.2018.09.003, indexed in Pubmed: 30360886.

23. Suárez de Lezo J, Martín P, Pan M, et al. Bioresorbable vascular scaffold for the treatment of coronary bifurcation lesions: immediate results and 1-year follow-up. Rev Esp Cardiol (Engl Ed). 2016; 69(6): 554-562, doi: 10.1016/j.rec.2015.12.004, indexed in Pubmed: 26949014.

24. Tanaka A, Jabbour RJ, Kawamoto H, et al. Preliminary report of clinical outcomes after single crossover bioresorbable scaffold implantation without routine side branch strut dilation. Catheter Cardiovasc Interv. 2016; 88(6): 865-870, doi: 10.1002/ccd.26586, indexed in Pubmed: 27219102. 
25. Tanaka A, Latib A, Kawamoto H, et al. Clinical outcomes following bifurcation double-stenting with bioresorbable scaffolds. Catheter Cardiovasc Interv. 2016; 88(6): 854-862, doi: 10.1002/ ccd.26579, indexed in Pubmed: 27184769.

26. Nishio S, Kosuga K, Igaki K, et al. Long-term ( $>10$ years) clinical outcomes of first-in-human biodegradable poly-l-lactic acid coronary stents: Igaki-Tamai stents. Circulation. 2012; 125(19): 2343-2353, doi: 10.1161/CIRCULATIONAHA.110.000901, indexed in Pubmed: 22508795.

27. Stone GW, Ellis SG, Gori T, et al. ABSORB IV Investigators. Blinded outcomes and angina assessment of coronary bioresorbable scaffolds: 30 -day and 1-year results from the ABSORB IV randomised trial. Lancet. 2018; 392(10157): 1530-1540, doi: 10.1016/S0140-6736(18)32283-9, indexed in Pubmed: 30266412.

28. Han Y, Xu Bo, Fu G, et al. A randomized trial comparing the neovas sirolimus-eluting bioresorbable scaffold and metallic everolimus-eluting stents. JACC Cardiovasc Interv. 2018; 11(3): 260-272, doi: 10.1016/j.jcin.2017.09.037, indexed in Pubmed: 29413240.
29. Ferenc M, Buettner HJ, Gick M, et al. Clinical outcome after percutaneous treatment of de novo coronary bifurcation lesions using first or second generation of drug-eluting stents. Clin Res Cardiol. 2016; 105(3): 230-238, doi: 10.1007/s00392-015-0911-7, indexed in Pubmed: 26329585.

30. Lee JM, Hahn JY, Kang J, et al. Differential prognostic effect between first- and second-generation drug-eluting stents in coronary bifurcation lesions: patient-level analysis of the korean bifurcation pooled cohorts. JACC Cardiovasc Interv. 2015; 8(10): 1318-1331, doi: 10.1016/j.jcin.2015.05.014, indexed in Pubmed: 26315734.

31. Muramatsu T, Onuma Y, García-García HM, et al. Incidence and short-term clinical outcomes of small side branch occlusion after implantation of an everolimus-eluting bioresorbable vascular scaffold: an interim report of 435 patients in the ABSORB-EXTEND single-arm trial in comparison with an everolimus-eluting metallic stent in the SPIRIT first and II trials. JACC Cardiovasc Interv. 2013; 6(3): 247-257, doi: 10.1016/j.jcin.2012.10.013, indexed in Pubmed: 23517836. 